\title{
Biopsia estereotáxica mamaria
}

\author{
Breast Stereotaxic Biopsy
}
Jennifer Richardson Maturana Vanessa Castaño Lopera Claudia Isabel Cabarcas Herrera' Luz Estella García
Eduardo de Núbila Lizcano Israel Díaz-Yunez

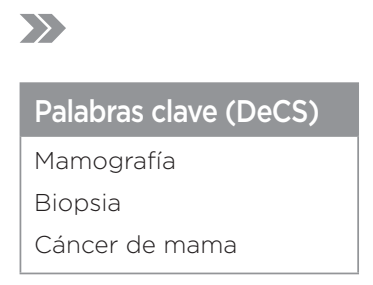

Key words (MeSH)

Mammography

Biopsy

Breast neoplasms
'Médica residente de Radiología e Imágenes Diagnósticas, Universidad del Norte. Barranquilla, Colombia.

2 Médica radióloga, SURA Ayudas Diagnósticas. Sede VIVA. Barranquilla, Colombia.

3Médico radiólogo, Centro de Imágenes Diagnósticas y Terapéuticas, CEDIUL, S. A. Barranquilla, Colombia.

${ }^{4}$ Coordinador médico e investigador Senior, CEDIUL, S. A. Barranquilla, Colombia.

\section{Resumen}

Objetivos: Caracterizar una cohorte de pacientes con cáncer de mama diagnosticadas por guía estereotáxica y confirmadas por patología. Describir las bondades del método desde el punto de vista de seguridad y ambulatorio, ayuda diagnóstica y guía para el cirujano oncólogo. Materiales $\boldsymbol{y}$ métodos: Estudio observacional descriptivo de cohorte retrospectiva. Se revisaron los registros de las pacientes que asistieron por sospecha de lesiones mamarias a quienes se les realizó una biopsia guiada por estereotaxia (BGE) entre mayo de 2016 y diciembre de 2017, con técnica mamográfica estándar. Se evaluó la distribución de las variables cuantitativas con el test de Kolmogorov-Smirnov. Se realizaron cruces exploratorios entre los hallazgos de la BGE y el diagnóstico patológico con el test Chi-cuadrado; para la comparación de variables cuantitativas por seguir una distribución no paramétrica se utilizó el test de $U$ de Mann Whitney. Resultados: Se incluyeron 36 mujeres con una mediana de edad de 56,5 años, el total de la muestra tuvo una clasificación radiológica prebiopsia BI-RADS 4b (27,8\%), seguido de BI-RADS 3 (25\%). Las lesiones más frecuentes de la BGE fueron microcalcificaciones (55,6 \%). La patología fue carcinoma in situ en un 47,2 \%. No se encontraron diferencias significativas en la distribución de edad por patología. Conclusiones: La biopsia de mama guiada por estereotaxia es un método seguro y confiable para el diagnóstico de pacientes con cáncer de mama, con una excelente correlación entre los hallazgos del BI-RADS y la patología, como guía para la intervención terapéutica del cirujano oncólogo.

\begin{abstract}
Summary
Objectives: Characterize a cohort of patients with breast cancer diagnosed by stereotaxic guidance, and confirmed by pathology. Describe the benefits of the method from the safety and ambulatory point of view, diagnostic help and guidance for the oncologist surgeon. Materials and methods: Descriptive observational study of a retrospective cohort. We reviewed all the records of patients who attended for suspected of breast lesions who underwent a biopsy guided by stereotaxis (BGS) in the period between May 2016 and December 2017, with standard mammography technique. The distribution of the quantitative variables was evaluated with the Kolmogorov-Smirnov test. Exploratory crosses were made between the findings of the BGS and the pathological diagnosis with the Chi-square test; for the comparison of quantitative variables following a nonparametric distribution the Mann Whitney $U$ test was used. Results: We included 36 women with a median age of 56.5 years, the total of the sample had a radiological classification pre-biopsy BIRADS 4b (27.8\%), followed by BIRADS 3 (25\%). The most frequent lesions described in the BGS were microcalcifications (55.6\%). The pathology was carcinoma in situ in $47.2 \%$. No significant differences were found in the age distribution by pathology ( $p$ = 0.109). Conclusions: Stereotactic-guided breast biopsy is a safe and reliable method for the diagnosis of patients with breast cancer, with an excellent correlation between the findings according to the BlRADS category and the pathology, as a guide for therapeutic intervention by the oncologist surgeon.
\end{abstract}

\section{Introducción}

El cáncer de mama es el más frecuente en la mujer, tanto en los países desarrollados, como en los países en desarrollo. En Colombia, en el periodo comprendido entre 2015-2016 ocupó el primer lugar entre los tumores malignos más frecuentes, con un total de 43.846 mujeres afectadas. Se presentaron 3.954 pacientes durante el periodo de análisis, lo cual representa una incidencia del $14 \%$. La mortalidad por esta patología fue de 2.055 pacientes (1).

La detección precoz sigue siendo la piedra angular del control del cáncer de mama, debido a que mejora el pronóstico y la supervivencia de las pacientes afectadas (2). Para la evaluación inicial de la mama se utiliza el examen clínico, la mamografía y la ultrasonografía $(3,4)$. Cuando se identifican microcalcificaciones en la mamografía sin lesión palpable, la biopsia guiada por estereotáxica (BGE), (SBB, por sus siglas en inglés de Stereotactic Breast Biopsy) puede establecer un diagnóstico (5-7). En esta investigación se destacan las características generales de la experiencia en la aplicación de este procedimiento en la institución en la que se hizo el estudio, en pacientes con dichas características, con diagnóstico histopatológico confirmatorio para cáncer de mama. 
Cuando se sospecha lesión, cualquiera que sea el método de imagen empleado, la indicación es practicar una biopsia (5,8-10). Generalmente, las anormalidades detectadas por mamografía corresponden a microcalcificaciones, masas o distorsiones de la arquitectura $(3,7)$. En estos casos los hallazgos de cáncer representan aproximadamente el $33 \%$ y la biopsia por estereotaxia constituye el procedimiento ideal $(5,6)$. En el resto de pacientes los hallazgos por biopsia serán benignos y, a excepción de la hiperplasia atípica, no requerirán intervenciones adicionales (3,11-13).

La biopsia guiada por estereotaxia utiliza la tecnología imagenológica para obtener muestras de lesiones sin necesidad de cirugía, no requiere anestesia general, ni hospitalización y ayuda a identificar el tipo histológico de la lesión para la planificación terapéutica $(5,13)$. Además, tiene un costo menor comparado con la biopsia por escisión, incluso, puede reemplazarla (7).

En las masas mamarias palpables, la BGE ayuda a proporcionar información prequirúrgica, histología, tinción inmunohistoquímica de estrógeno y receptores de progesterona, expresión de HER2, y puede ser útil en la planificación del procedimiento quirúrgico $(5,6)$.

En la experiencia del equipo multidisciplinario, se ha demostrado este postulado: En alteraciones mamográficas de lesiones sospechosas, como las microcalcificaciones, en las cuales no se demuestra adecuada representación en la evaluación ecográfica, es limitada la guía de la toma de muestra por este método (14), así la posibilidad de contar con el equipo para realizar las biopsias de mama guiadas por estereotaxia, hace posible la derivación hacia esta técnica.

\section{Materiales y métodos}

Estudio observacional descriptivo de cohorte retrospectiva. Se revisaron todos los registros de los pacientes que asistieron por sospecha de lesiones mamarias ocultas o palpables a quienes se les realizó una BGE en el período comprendido entre mayo de 2016 y diciembre de 2017. Todas las biopsias se tomaron bajo guía por estereotaxia, se extrajo un promedio de 6 muestras para estudio histopatológico. Durante el periodo evaluado se realizaron 342 biopsias guiadas por estereotaxia. Se aplicaron criterios de inclusión y exclusión para una muestra final utilizada para el análisis de 36 pacientes. Las variables cuantitativas se describieron como medianas y rango intercuartílico para su análisis, para las variables cualitativas se calcularon frecuencias y porcentajes, los resultados se presentaron como gráficos y tablas de distribución. Se realizaron comparaciones entre los hallazgos de la biopsia guiada por estereotaxia y el diagnóstico patológico con el test Chi-cuadrado, para la comparación de variables cuantitativas por seguir una distribución no paramétrica se utilizó el test de U de Mann Whitney.

\section{Resultados}

Se incluyeron 36 mujeres con una mediana de edad de 56,5 (RIQ $51,5-63,0)$ años, el total de la muestra tuvo un diagnóstico maligno confirmado por patología, en su mayoría con una clasificación radiológica prebiopsia BI-RADS 4b (27,8 \%), seguido de BI-RADS 3 (25\%). Se encontraron frecuencias similares en la localización de las lesiones, en el seno derecho $50,0 \%$ y el izquierdo $47,2 \%$, afectando principalmente el cuadrante superior externo 41,7\% (tabla 1). Las lesiones más frecuentemente descritas en la BGE fueron microcalcificaciones (55,6\%), seguidas de asimetría (tabla 1 y figura 1). Con respecto al resultado de la patología, el 47,2\% de los casos correspondieron a carcinoma in situ, seguido del carcinoma canalicular, con un 25,0\% (tabla 1 y figura 2). Las microcalcificaciones fueron el hallazgo más frecuente en la biopsia guiada por estereotaxia entre las pacientes a quienes se les diagnosticó por patología carcinoma in situ, canalicular y ductal (15); mientras que la asimetría se presentó con mayor frecuencia entre las pacientes con carcinoma infiltrante; sin embargo, estas diferencias no fueron estadísticamente significativas $(p=0,501)$ (tabla 2$)$. Al evaluar la distribución de edad por BI-RADS se encontró que las mujeres con edad más avanzada presentaron BI-RADS 4; no obstante, estas diferencias no fueron estadísticamente significativas $(p=0,909)$ (figura 3 ). No se encontraron diferencias significativas en la distribución de edad por patología $(p=0,109)$ (figura 4).

\section{Tabla 1. Características generales de la muestra}

\begin{tabular}{|c|c|c|}
\hline \multicolumn{2}{|r|}{ Edad, años } & $\begin{array}{c}56,5 \\
(51-630)\end{array}$ \\
\hline \multirow{3}{*}{ Seno } & Derecho & $18(50,0)$ \\
\hline & Izquierdo & $17(47,2)$ \\
\hline & Bilateral & $1(2,8)$ \\
\hline \multirow{6}{*}{ Cuadrante } & Cuadrante superior externo & $15(41,7)$ \\
\hline & Cuadrante inferior interno & $9(25,0)$ \\
\hline & Cuadrante superior interno & $4(11,1)$ \\
\hline & Retroareolar & $4(11,1)$ \\
\hline & Cuadrante externo & $2(5,6)$ \\
\hline & Cuadrante inferior externo & $2(5,6)$ \\
\hline \multirow{6}{*}{ BI-RADS } & 3 & $9(25,0)$ \\
\hline & 4 & $3(8,3)$ \\
\hline & $4 \mathrm{~A}$ & $4(11,1)$ \\
\hline & 4B & $10(27,8)$ \\
\hline & $4 C$ & $4(11,1)$ \\
\hline & 5 & $6(16,7)$ \\
\hline \multirow{4}{*}{$\begin{array}{l}\text { Diagnóstico } \\
\text { patológico }\end{array}$} & In situ & $17(47,2)$ \\
\hline & Canalicular & $9(25,0)$ \\
\hline & Ductal & $7(19,4)$ \\
\hline & No especial & $3(8,3)$ \\
\hline \multirow{10}{*}{$\begin{array}{l}\text { Hallazgos } \\
\text { BGE }\end{array}$} & Microcalcificaciones & $20(55,6)$ \\
\hline & Asimetría & $5(13,9)$ \\
\hline & Asimetría con microcalcificaciones & $3(8,3)$ \\
\hline & Microcalcificaciones agrupadas & $2(5,6)$ \\
\hline & Distorsión con calcificación & $1(2,8)$ \\
\hline & Ganglio axilar y microcalcificaciones & $1(2,8)$ \\
\hline & Nódulo con microcalcificaciones & $1(2,8)$ \\
\hline & $\begin{array}{l}\text { Microcalcificaciones, distorsión de la } \\
\text { arquitectura, espiculado }\end{array}$ & $1(2,8)$ \\
\hline & Nódulo & $1(2,8)$ \\
\hline & Nódulo irregular & $1(2,8)$ \\
\hline
\end{tabular}


Tabla 2. Diagnóstico patológico vs. hallazgos BGE

\begin{tabular}{|c|c|c|c|c|c|c|}
\hline & \multicolumn{4}{|c|}{ Diagnóstico patológico } & \multirow[b]{2}{*}{ valor $p$} \\
\hline & & In situ & $\begin{array}{c}\text { No } \\
\text { especial }\end{array}$ & Canalicular & Ductal & \\
\hline \multirow{10}{*}{ Hallazgos BGE } & Asimetría & $1(2,8)$ & $2(5,6)$ & $1(2,8)$ & $1(2,8)$ & \multirow{10}{*}{0,501} \\
\hline & $\begin{array}{c}\text { Asimetría con } \\
\text { microcalcificaciones }\end{array}$ & $2(5,6)$ & $O(O)$ & $1(2,8)$ & $\mathrm{O}(\mathrm{O})$ & \\
\hline & $\begin{array}{l}\text { Distorsión con } \\
\text { calcificación }\end{array}$ & $\mathrm{O}(\mathrm{O})$ & $O(O)$ & $1(2,8)$ & $O(O)$ & \\
\hline & $\begin{array}{c}\text { Ganglio axilar y } \\
\text { microcalcificaciones }\end{array}$ & $1(2,8)$ & $\mathrm{O}(\mathrm{O})$ & $O(0)$ & $\mathrm{O}(\mathrm{O})$ & \\
\hline & $\begin{array}{l}\text { Nódulo con } \\
\text { microcalcificaciones }\end{array}$ & $\mathrm{O}(\mathrm{O})$ & $\mathrm{O}(\mathrm{O})$ & $1(2,8)$ & $\mathrm{O}(\mathrm{O})$ & \\
\hline & $\begin{array}{l}\text { Microcalcificaciones, } \\
\text { distorsión de la } \\
\text { arquitectura, espiculado }\end{array}$ & $\mathrm{O}(\mathrm{O})$ & $\mathrm{O}(\mathrm{O})$ & $\mathrm{O}(\mathrm{O})$ & $1(2,8)$ & \\
\hline & Microcalcificaciones & $11(30,6)$ & $1(2,8)$ & $4(11,1)$ & $4(11,1)$ & \\
\hline & $\begin{array}{c}\text { Microcalcificaciones } \\
\text { agrupadas }\end{array}$ & $1(2,8)$ & $O(O)$ & $1(2,8)$ & $\mathrm{O}(\mathrm{O})$ & \\
\hline & Nódulo & $\mathrm{O}(\mathrm{O})$ & $\mathrm{O}(\mathrm{O})$ & $\mathrm{O}(\mathrm{O})$ & $1(2,8)$ & \\
\hline & Nódulo irregular & $1(2,8)$ & $O(0)$ & $O(O)$ & $O(O)$ & \\
\hline
\end{tabular}

Figura 1. Distribución de hallazgos BGE

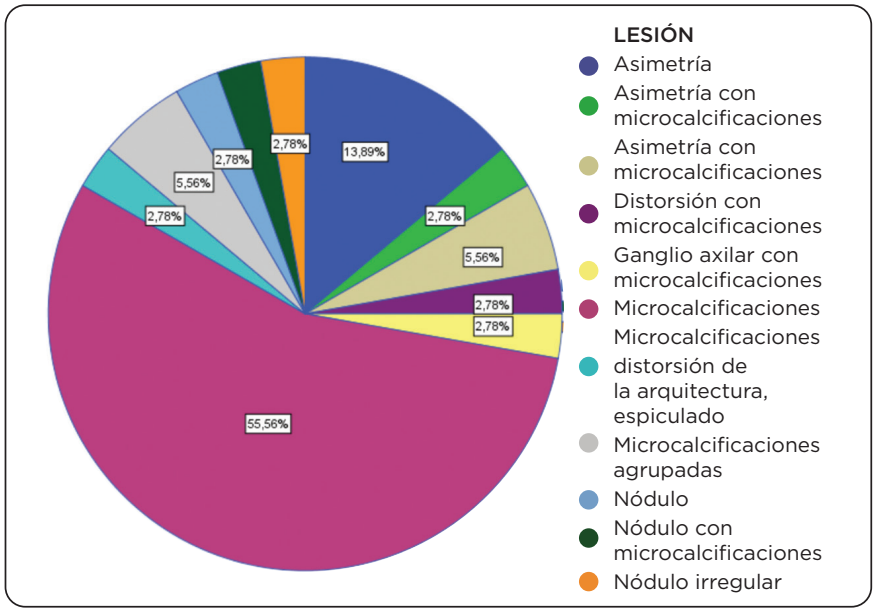

Figura 3. Distribución de edad por clasificación BI-RADS

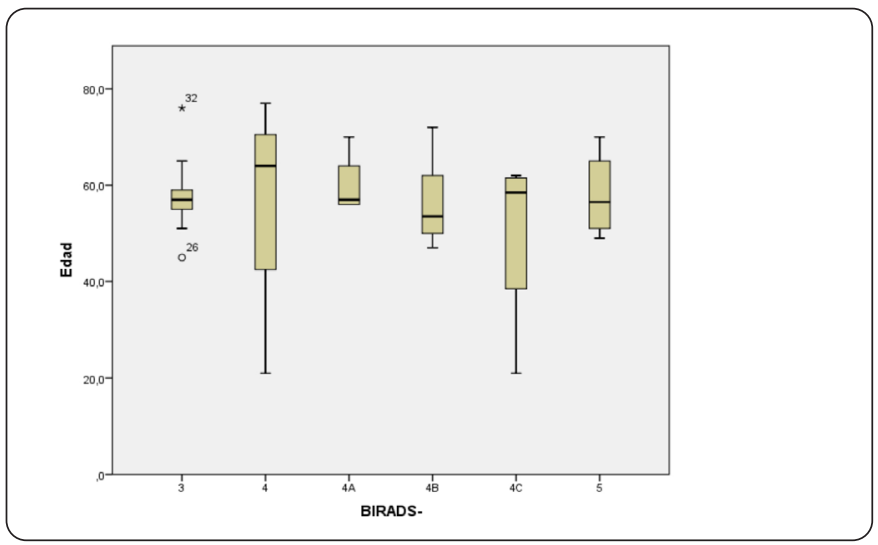

Kruskal Wallis $p=0,909$
Figura 2. Distribución de hallazgos patológicos

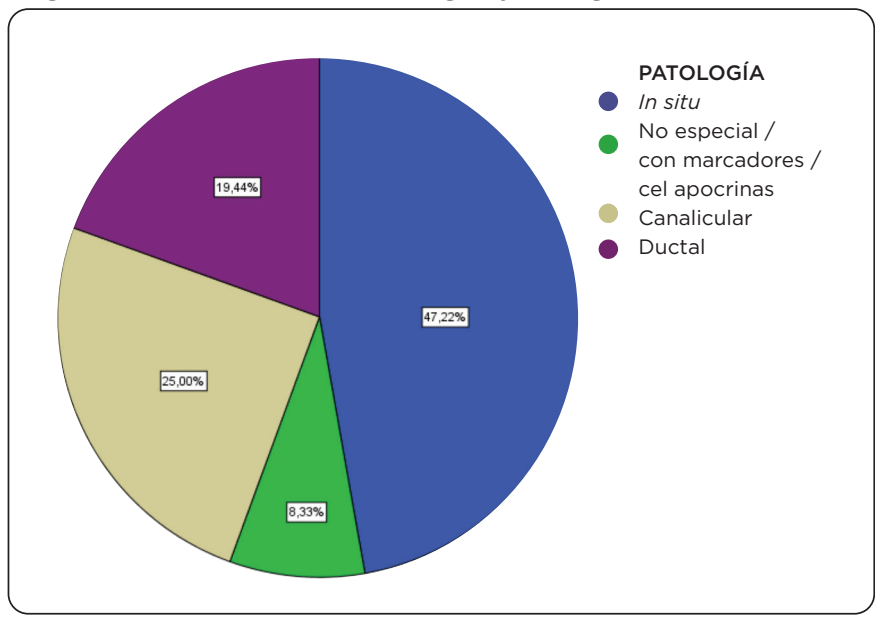

Figura 4. Distribución de edad por patología

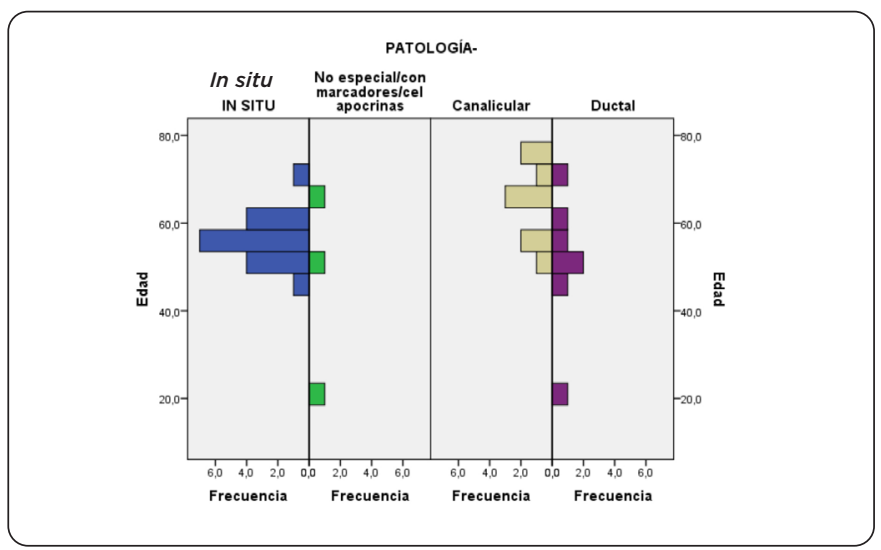

Kruskal Wallis $p=0,109$ 


\section{Discusión}

El cáncer de mama es una patología frecuente y con una tasa de mortalidad alta si no se diagnostica en etapas tempranas. Para ello, la mamografía ha intervenido de manera positiva y en conjunto con la clasificación BI-RADS se pueden detectar lesiones sugestivas de neoplasias que requieren biopsia. La BGE se encuentra entre las técnicas utilizadas para la toma de muestras de calcificaciones observadas en mamografía sin lesión palpable con una excelente concordancia del $96 \%$ entre el estudio imagenológico previo y el estudio histopatológico de la muestra obtenida por esta técnica (15). En esta cohorte el total de la muestra tuvo un diagnóstico maligno confirmado por patología, pero fue prevalente el carcinoma in situ encontrado en un $47,2 \%$ de los casos, lo que indica una buena correlación entre los hallazgos mamográficos, la muestra obtenida bajo guía estereotáxica y la patología, lo cual es coherente con lo expuesto en la literatura.

\section{Conclusión}

La biopsia de mama guiada por estereotaxia es un método seguro y confiable que localiza con precisión geométrica las lesiones, para el diagnóstico de pacientes con cáncer de mama, con una excelente correlación entre los hallazgos del BI-RADS y la patología, como guía para la intervención terapéutica del cirujano oncólogo.

\section{Referencias}

1. Siegel RL, Miller KD, Jemal A. Cancer statistics, 2017. CA Cancer J Clin [Internet]. 2017;67(1):7-30. [citado 2020 jul. 19]. http://dx.doi.org/10.3322/caac.21387

2. WHO. Breast cancer. WHO [Internet]. 2018 [citado 2020 jul. 19]. Disponible en: https:/www.who.int/cancer/prevention/diagnosis-screening/breast-cancer/en/

3. McDonald ES, Clark AS, Tchou J, Zhang P, Freedman GM. Clinical diagnosis and management of breast cancer. J Nucl Med [Internet]. [citado 2020 jul. 19]. 2016;57(Suppl 1):9s-16s.. http://dx.doi.org/10.2967/jnumed.115.157834

4. Kolb TM, Lichy J, Newhouse JH. Comparison of the performance of screening mammography, physical examination, and breast US and evaluation of factors that influence them: An analysis of 27,825 patient evaluations. Radiology [Internet]. 2002 [citado 2020 jul. 19];225(1):165-75. Disponible en: https://pubs.rsna.org/doi/abs/10.1148/ radiol.2251011667

5. Cangiarella J, Waisman J, Symmans WF, Gross J, Cohen JM, Wu H, et al. Mammotome core biopsy for mammary microcalcification: analysis of 160 biopsies from 142 women with surgical and radiologic followup. Cancer [Internet]. 2001[citado 2020 jul. 19]. 2001;91(1):173-7. Disponible en: http://dx.doi.org/

6. Margolin FR, Kaufman L, Jacobs RP, Denny SR, Schrumpf JD. Stereotactic core breast biopsy of malignant calcifications: Diagnostic yield of cores with and cores without calcifications on specimen radiographs. Radiology [Internet]. 2004 [cited 2020 jul. 19];233(1):251-4. Disponible en: https://pubs.rsna.org/doi/abs/10.1148/ radiol.2331031680

7. Liberman L. Percutaneous image-guided core breast biopsy. Radiol Clin North Am [Internet]. 2002;40(3):483-500, vi. [citado 2020 jul. 19]. Disponioble en: http://dx.doi. org/

8. Verkooijen HM. Diagnostic accuracy of stereotactic large-core needle biopsy for nonpalpable breast disease: Results of a multicenter prospective study with $95 \%$ surgical confirmation. Int J Cancer [Internet]. 2002 [citado 2020 jul 19];99(6):853-9. Disponible en: https://onlinelibrary.wiley.com/doi/full/10.1002/ijc.10419

9. Hoda SA, Rosen PP. Practical Considerations in the Pathologic Diagnosis of Needle Core Biopsies of Breast [Internet]. [cited 2020 Jul 19]. Am J Clin Pathol. 2002;118(1):101-8. Disponible en: https://academic.oup.com/ajcp/article-abstract/118/1/101/1758427

10. Kettritz U, Rotter K, Schreer I, Murauer M, Schulz-Wendtland R, Peter D, et al. Stereotactic vacuum-assisted breast biopsy in 2874 patients: A multicenter study. Cancer [Internet]. 2004 Jan 15 [cited 2020 jul 19];100(2):245-51. Disponible en: https://pubmed.ncbi.nlm.nih.gov/14716757/

11. Calhoun BC, Collins LC. Recommendations for excision following core needle biopsy of the breast: a contemporary evaluation of the literature. Histopathology [Internet]. [citado 2020 jul 19]2016;68(1):138-51. Disponible en: http://dx.doi.org/10.1111/ his. 12852

12. Mooney KL, Bassett LW, Apple SK. Upgrade rates of high-risk breast lesions diagnosed on core needle biopsy: A single-institution experience and literature review [Internet].
Modern Pathology. [cited 2020 jul 19]. 2016;29(12):1471-84. Disponible en: https:// pubmed.ncbi.nlm.nih.gov/27538687/

13. Monticciolo DL, Hajdik RL, Hicks MG, Winford JK, Larkin WR, Vasek JV, et al Six-month short-interval imaging follow-up for benign concordant core needle biopsy of the breast: Outcomes in 1444 cases with long-term follow-up. Am J Roentgenol [Internet]. 2016 Oct 1 [citado 2020 jul 19];207(4):912-7. Disponible en: https:// pubmed.ncbi.nlm.nih.gov/27340732/

14. Díaz Yunez IJ, Parra Anaya GDJ. Microcalcificaciones detectadas en pacientes atendidos en el Centro de Diagnóstico de Ultrasonografico CEDIUL de la ciudad de Barranquilla durante el periodo Julio-Noviembre de 2012. Rev Salud Caribe. 2013;1:22-5.

15. Han BK, Choe YH, Ko YH, Nam SJ, Kim JH, Yang JH. Stereotactic core-needle biopsy of non-mass calcifications: Outcome and accuracy at long-term follow-up. Korean J Radiol [Internet]. 2003;4(4):217-23. Disponible en: http://dx.doi.org/10.3348/ kjr.2003.4.4.217

\section{Correspondencia}

Jennifer Richardson Maturana

Carrera 45 \# 79-96, apto. 503

Barranquilla, Colombia

jrichardson@uninorte.edu.co

Recibido para evaluación: 01 de julio de 2020 Aceptado para publicación: 21 de septiembre de 2020 